\title{
Médiévales
}

Langues, Textes, Histoire

54 | printemps 2008

Frères et sœurs

\section{Elsa MARMURSZTEJN, L'Autorité des maîtres. Scolastique, normes et société au XIII siècle, Paris, Les Belles}

Lettres, 2007, $431 \mathrm{p}$.

\section{Claire Angotti}

\section{(2) OpenEdition}

Journals

Édition électronique

URL : https://journals.openedition.org/medievales/5273

DOI : 10.4000/medievales.5273

ISSN : $1777-5892$

\section{Éditeur}

Presses universitaires de Vincennes

\section{Édition imprimée}

Date de publication : 1 juin 2008

Pagination : 159-162

ISBN : 978-2-84292-217-7

ISSN : 0751-2708

\section{Référence électronique}

Claire Angotti, "Elsa maRmURSzTEJn, L'Autorité des maitres. Scolastique, normes et société au XIII siècle, Paris, Les Belles Lettres, 2007, 431 p. », Médiévales [En ligne], 54 I printemps 2008, mis en ligne le 23 octobre 2008, consulté le 24 avril 2022. URL : http://journals.openedition.org/medievales/5273 ; DOI : https://doi.org/10.4000/medievales.5273

Ce document a été généré automatiquement le 24 avril 2022

Tous droits réservés 


\title{
Elsa MARMURSZTEJN, L'Autorité des
} maîtres. Scolastique, normes et société au XIII ${ }^{e}$ siècle, Paris, Les Belles Lettres, 2007, $431 \mathrm{p}$.

\author{
Claire Angotti
}

1 Le livre d'Elsa Marmursztejn est issu d'une thèse de doctorat soutenue en 1999 à l'École des Hautes Études en Sciences Sociales. Cet ouvrage n'est pas la simple publication du travail de doctorat mais en est une synthèse, dense et mûrie. Il se fonde sur la connaissance approfondie et la maîtrise de sources complexes, parfois inédites, longtemps négligées par les historiens : les quodlibeta. Il s'agit d'une forme de dispute, d'un exercice magistral développé par l'Université au XIII' siècle, organisé pendant deux périodes de l'année universitaire. Le problème auquel le maitre a à répondre est soulevé par un auditeur de la dispute, par toute personne (quilibet) souhaitant y assister : la questio peut porter sur n'importe quel sujet (de quolibet).

2 E. Marmursztejn étudie les questions quodlibétiques de quatre maitres qui semblent avoir eu une prédilection pour cet exercice : un dominicain, Thomas d'Aquin $(\dagger 1274)$ et trois séculiers, Gérard d'Abbeville (†1272), Henri de Gand ( $† 1293)$, Godefroid de Fontaines ( $†$ ca. 1306-1309), deux générations de théologiens qui ont enseigné à Paris. L'auteur fait aussi référence à d'autres œuvres de ces maîtres, quand elles éclairent leurs quodlibeta, à des ouvrages qui ont alimenté leurs réflexions (commentaires des Sentences de Bonaventure et Richard de Mediavilla, la Summa aurea de Guillaume d'Auxerre, les quodlibeta de Servais du Mont-Saint-Éloi, etc.)

3 E. Marmursztejn étudie de manière magistrale, au travers des quodlibets variés qui leur ont été posés, la manière dont les maîtres ont affirmé leur autorité et proposé des normes à l'ensemble de la société. Parallèlement à ce premier objectif, l'auteur développe une réflexion sur le fonctionnement du quodlibet dans l'élaboration de cette norme. Ainsi elle démontre en quoi ces "questions-limites" que sont les quodlibeta proposés à la rationalité et à l'analyse sont, pour les maîtres, l'occasion d'embrasser des 
champs problématiques vastes. Chaque chapitre est ainsi fondé sur l'analyse d'une (série de) question(s) quodlibétique(s) : l'auteur en expose les enjeux doctrinaux et intellectuels et met en rapport la questio avec son contexte politique et social.

4 L'ouvrage est structuré en trois grandes parties : la première (p. 19-82) traite de l'image de la fonction intellectuelle et de la construction de l'autorité des maîtres. L'auteur s'intéresse à la manière dont ils se présentent comme élite intellectuelle (chapitre 1). Ce n'est qu'à la fin du XIII ${ }^{e}$ siècle que les maîtres s'affirment comme élite sociale en proposant un schéma tripartite affirmant la puissance et l'autorité de leur activité intellectuelle (le studium) face à l'autorité ecclésiastique (sacerdotium) et laïque (regnum). L'étude des quodlibeta permet à l'auteur d'analyser la réflexion des maîtres sur leur pratique et leur responsabilité sociale. Tous les théologiens insistent sur l'acte d'enseigner, sur l'importance de l'effort de transmission, n'hésitant pas, à l'instar d'Henri de Gand, à affirmer que la fonction (d'enseignant) primait le titre (de maître). Les quodlibets sont la manifestation de ce souci pédagogique et doctrinal.

5 De fait, dans leurs quodlibeta, les théologiens affirment qu'ils ont seuls compétence à former les gens d'Église justifiant, ainsi une forme de leur responsabilité sociale (chapitre 2). Par delà les différences et les controverses, maîtres séculiers comme mendiants sont attachés à l'affirmation de la supériorité de la science théologique, susceptible de former, du fait de leur activité d'enseignement, l'ensemble des cadres de l'Église, mediocres ( « intellectuels intermédiaires », tels que les prédicateurs) et par leur biais, les minores (prêtres de paroisses). Cette « aristocratie du savoir » emploie l'image aristotélicienne de l'architecte pour souligner la suprématie de la fonction intellectuelle et spéculative sur la fonction pastorale. L'ambition des théologiens est donc vaste : il s'agit rien moins que d'établir des principes que d'autres mettraient en pratique, non seulement au profit de leurs élèves et de leurs confrères ou au profit de l'institution ecclésiale mais aussi (et surtout) au profit de la société toute entière.

L'enseignement des maîtres s'est cependant heurté à un certain nombre de limites (chapitre 3). Les disputes quodlibétiques ont été l'occasion de réfléchir à la nécessité, parfois, de taire ou de rétracter leur enseignement. Plusieurs quodlibeta ayant pour sujet le mensonge ou les « vaines paroles » ont été l'occasion de développer le thème du crédit à accorder à la parole magistrale. Dans ce chapitre, E. Marmursztejn analyse aussi les rapports complexes entretenus par les maîtres et les puissants. Les maîtres (notamment Henri de Gand, Godefroid de Fontaines), conscients des enjeux dont ils étaient l'objet, se posent de fait comme "docteurs de la vérité " responsables de la collectivité chrétienne. Ils revendiquent une liberté intellectuelle afin d'élaborer une communis opinio magistrorum qui doit se développer au-delà du monde des écoles.

7 Ces trois chapitres constituent le cadre indispensable pour saisir les deux parties suivantes qui traitent ainsi de l'ambition normative des théologiens (p. 83-150) puis des normes qu'ils proposent à la société chrétienne (p. 151-264).

8 Le chapitre 4 inscrit la réflexion normative des théologiens dans le contexte de l'élaboration des normes laïques, ecclésiastiques qui marquent les $\mathrm{XII}^{\mathrm{e}}$-XIII ${ }^{\mathrm{e}}$ siècles. La norme voulue par les théologiens s'inscrit dans un cadre chrétien, constitué par la loi nouvelle que les théologiens considèrent comme donnée par le Christ. Toute loi humaine est ainsi le résultat de l'interprétation progressive qu'ils conduisent. L'auteur décrit, de manière synthétique, la mise en place de différentes normes (laïque, ecclésiastique) et leur très grande diversité. Se manifeste ainsi la normativité issue du 
prince législateur et ses principaux développements, ainsi que la normativité liée à l'affirmation des droits savants.

9 Le chapitre 5 porte sur les quodlibeta ayant conduit les maîtres à évaluer les normes et les pouvoirs normatifs ecclésiastiques. Les sujets abordés par les quodlibeta sont très variés. Leur point commun est de conduire les maîtres à définir autorité pontificale comme autorité épiscopale. Ces questions posées aux maîtres, ces "cas limite» nécessitent de leur part une définition des notions qu'ils emploient tout comme la justification de leur intervention vis-à-vis des diverses autorités ecclésiastiques.

Le chapitre 6 correspond à l'évaluation de la norme et des pouvoirs laïcs par ces mêmes théologiens. E. Marmursztejn s'appuie sur l'analyse rigoureuse des solutions proposées par les maîtres à des questions quodlibétiques très variées, et s'attache aussi à leurs méthodes de démonstration. Est essentiellement visé le pouvoir princier au travers de questiones touchant la capacité à légiférer. Ce thème, au cœur des préoccupations des hommes du XIII ${ }^{e}$ siècle, est à relier à la construction du pouvoir étatique qui doit, selon les maitres, être ordonné au bien commun. Les théologiens n'hésitent d'ailleurs pas à poser les limites de l'autorité du prince. En insistant sur la science nécessaire au bon prince et sur l'obligation qu'il a de justifier sa législation (notamment fiscale), les maitres s'offrent en modèles: le pouvoir normatif du prince doit être bâti sur une science et une pédagogie.

11 Souverain pontife, évêques, princes, auteurs de la norme, comme prêtres, moniales, contribuables, juifs, sujets de la norme, sont l'objet de la pensée et de l'exercice de la normativité théologiques. Les maîtres sont donc conduits à proposer des normes à l'ensemble de la société chrétienne, normes qu'étudie E. Marmursztejn dans la troisième partie de son ouvrage.

12 Les droits portant sur les biens et les revenus ecclésiastiques sont l'objet du chapitre 7. Plusieurs questions quodlibétiques touchent en effet à la dîme et à la question, vitale pour les maîtres séculiers, du cumul des bénéfices. Les quatre maitres dont $\mathrm{E}$. Marmursztejn étudie l'œuvre quodlibétique se sont penchés sur la dîme. La permanence des questiones posées sur ce sujet trahit un contexte où sa perception, exclusive à l'Église, était très contestée. E. Marmursztejn se livre à une comparaison des méthodes comme des solutions magistrales: malgré des divergences, leur point commun est, tout en s'appuyant sur le droit canon, de s'affranchir de ce dernier et de proposer des solutions qui ne sont pas celles des juristes, affirmant, une nouvelle fois, le caractère ancillaire du droit. Quant à la question portant sur les bénéfices, les maitres sont unanimes sur les critères qui doivent prévaloir : tout à la fois l'utilité de l'Église et les compétences de ses ministres. Au-delà d'un argumentaire pro domo, les séculiers insistent sur la valeur du desservant et justifient, outrepassant ainsi le droit canon, le cumul des bénéfices.

13 Le chapitre 8 prolonge le thème de l'acquisition et des échanges de biens qui concernent surtout les laïcs. Les questions économiques entrent effectivement dans le champ de la pensée scolastique : se forme, sous l'influence de la théologie morale, une sorte d'«éthique économique ». Ce sont les contrats de rentes qui sont l'objet de l'analyse des théologiens : ils donnent lieu à d'âpres débats. Cela permet aux maîtres d'élaborer non seulement des solutions originales (par exemple sur le rôle et la fonction de l'argent) mais aussi de s'interroger, par delà la réflexion sur les biens, sur le statut des personnes. 

promu par les théologiens (chapitre 9). L'originalité de la pensée des maîtres tient au fait qu'ils modèlent le droit des personnes sur celui des biens. Une fois encore, une série de questions, qui peut paraître hétéroclite, permet de saisir l'effort normatif des maîtres concernant les droits personnels (obligation créée par des vœux, baptême forcé des enfants juifs etc.). Toutes ces questiones et les réponses nuancées qu'ont proposées les maîtres démontrent qu'ils accordaient une place essentielle aux relations entre les personnes.

C'est pour cette raison que l'ouvrage s'achève par un chapitre consacré à la question de la responsabilité personnelle (chapitre 10). Plusieurs questiones traitées par Henri de Gand, permettent au maître de développer très finement et de manière extrêmement novatrice une pensée axée sur l'intention personnelle, mise en relation avec la normativité pénale. Le maître met l'accent sur les droit individuels, insistant sur l'idée que la personne a un droit de propriété sur elle-même, idée dont certains philosophes $\mathrm{du} \mathrm{XVII}^{\mathrm{e}}$ siècle seront les héritiers. On saisit l'ambition universaliste de la norme universitaire : les théologiens dépassent le droit positif et proposent ainsi à l'ensemble de la société une norme élaborée, malgré des divergences d'opinion et des controverses, selon des méthodes et des valeurs communes.

Les théologiens ne se sont donc pas coupés des réalités, des enjeux sociaux: E. Marmursztejn en conclut que cette attitude est la preuve qu'ils se pensent comme un «troisième pouvoir", attitude qui justifie l'engagement de ces intellectuels dans le monde pratique.

17 Jusqu'alors peu de travaux démontraient l'extraordinaire fécondité de la dispute quodlibétique, se cantonnant, pour l'essentiel, à des présentations doctrinales de la pensée de tel ou tel maître (c'est ce qui a souvent été fait pour Thomas d'Aquin ou Henri de Gand), en liant quodlibet et querelle entre séculiers et mendiants (pensons aux travaux d'Y.-M. Dufeil), en décrivant strictement le genre littéraire spécifique que constitue la dispute quodlibétique (comme l'ont fait les pionniers que sont A. Landgraf et P. Glorieux, ou, plus récemment, J.F. Wippel). E. Marmursztejn renouvelle entièrement la manière d'employer les sources quodlibétiques. Sans rien céder aux enjeux doctrinaux qui sous-tendent les questions qui sont posées aux maîtres, elle démontre l'importance du quodlibet dans l'élaboration de la pensée scolastique.

Accompagné d'une bibliographie et d'un index des noms de personnes (on regrettera toutefois l'absence d'un index portant sur les concepts employés par les maitres) l'ouvrage d'E. Marmursztejn riche, érudit, s'inscrit au confluent de l'histoire des universités (J. Verger) et de l'histoire des intellectuels qui les peuplent, groupe social dont l'étude a été lancée par J. Le Goff en 1957, étude à laquelle E. Marmursztejn contribue magistralement ; au confluent aussi des travaux portant sur le maniement du savoir (O. Weijers) et sur l'anthropologie scolastique (A. Boureau), L'autorité des maitres illustre la complexité de l'identité des intellectuels, la haute idée qu'ils se faisaient de leur fonction sociale, leurs efforts pour la mettre en pratique dès le xIII ${ }^{e}$ siècle. 